\title{
First Order Phase Transition in the Plane Rotator Ferromagnetic Model in Two Dimensions
}

\author{
H. Kunz ${ }^{\star}$ \\ Laboratoire de Physique Théorique, Ecole Polytechnique Fédérale, CH-1001 Lausanne, Switzerland \\ C.-E.Pfister \\ Zentrum für Interdisziplinäre Forschung, Universität Bielefeld, D-4800 Bielefeld, \\ Federal Republic of Germany
}

\begin{abstract}
We show that the two-dimensional isotropic ferromagnetic rotator model exhibits a first order phase transition if the interaction decays as $r^{-\alpha}$ with $2<\alpha<4$.
\end{abstract}

\section{Introduction}

It is known that the isotropic two-dimensional ferromagnetic Heisenberg model does not exhibit spontaneous magnetization in two dimensions if the forces between the spins are not too long ranged. Typically for a potential of the type $J(r) \sim(r+1)^{-\alpha}$, where $r$ is the distance between the two spins in interaction, we need $\alpha>2$ in order to obtain a normal thermodynamic behaviour and $\alpha>4$ for the absence of spontaneous magnetization (Mermin and Wagner [1], Ruelle [2]. This result equally holds for a variety of classical spin systems, most notably the plane rotator and the classical Heisenberg model (Mermin [3], Vuillermot, Romerio [4], Dobrushin, Shlosman [5]). These proofs have put on a firm ground already existing intuitive arguments based on the droplet model of condensation (Fisher [6], Mermin [7]). These arguments are based on the fact that in order to create a droplet $D$ of size $L$ of the opposite phase, one needs an energy of the order of $\sum_{r \in D} r^{2} J(r)$ at worst. Therefore, if $J(r) \sim r^{-\alpha}, r$ being large, we obtain three different cases depending on the value of $\alpha$. If $\alpha>4$, the quantity $\sum_{r \in D} r^{2} J(r)$ is always bounded by a number independent from the size of $D$ and thus making big droplets very probable. Whereas if $\alpha \leqq 4$,

$$
\begin{aligned}
& \sum_{r \in D} r^{2} J(r) \sim L^{4-\alpha}, \quad \alpha<4, \\
& \sum_{r \in D} r^{2} J(r) \sim \ln L, \quad \alpha=4 .
\end{aligned}
$$

Therefore the energy of a droplet increases with the size of the droplet if $\alpha \leqq 4$, but as a power law when $\alpha<4$ and only logarithmically when $\alpha=4$. Such big droplets are very unlikely and therefore the order in the system cannot be destroyed, at least when $\alpha<4$. The case $\alpha=4$ is evidently more delicate. This situa-

^ Work supported by "Fonds National Suisse de la Recherche Scientifique". 
tion is very reminiscent of what happens in a one-dimensional system like the Ising model with long-ranged interaction. In fact the parallel intuitive argument had been put forward by Landau and developed by Thouless [8]. Since then Dyson was able to give a rigorous proof of the existence of a phase transition for a class of long-ranged potentials decaying for example like $r^{-\alpha}$ with $1<\alpha<2$ [9]. The strategy of his proof is the following: define an auxialiary model, which he calls hierarchical, for which the existence of long-range order is established and then compare the order parameter of this model to that of the original one by means of Griffiths inequalities. This is exactly the path we have followed, but by necessity we needed to restrict ourselves to plane rotators because it is the only model with a continuous internal symmetry group for which a useful analogue of Griffiths inequalities was proved by Ginibre [11].

Hence we see that, in the case of rotators at least, the intuitive arguments based on the droplet model lead to a correct conclusion as regard the absence or presence of long-range order. The borderline case $\alpha=4$ has been left undecided. This case merits attention because it would be very interesting to prove whether a Thouless effect, i.e. a jump in the spontaneous magnetization at the critical point $([8,10])$, occurs as it does in the one-dimensional case. However, we will not discuss this problem which is much more difficult.

\section{Description of the Model}

Let us consider the lattice $\mathbb{Z}^{2}$ which is formed by all linear combinations with integer coefficients of the two vectors $\boldsymbol{e}_{1}=(1,0)$ and $\boldsymbol{e}_{2}=(0,1)$. With each site $\boldsymbol{x}=\left(x_{1}, x_{2}\right)$ of $\mathbb{Z}^{2}$ we associate a continuous spin $\sigma(\boldsymbol{x})$ which is represented by a unit vector in $\mathbb{R}^{2}$. The plane rotator model, called hereafter model $\mathrm{I}$, is defined as follows: Let us consider a finite set $\Lambda$ of $\mathbb{Z}^{2}$. The hamiltonian is then

$$
H=-\sum_{\substack{\boldsymbol{x} \neq \boldsymbol{y} \\ \boldsymbol{x}, \boldsymbol{y} \in \Lambda}} J(|\boldsymbol{x}-\boldsymbol{y}|) \sigma(\boldsymbol{x}) \cdot \sigma(\boldsymbol{y})-h \sum_{\boldsymbol{x} \in \Lambda} \sigma(\boldsymbol{x})
$$

where $\sigma(\boldsymbol{x}) \cdot \sigma(\boldsymbol{y})$ means the scalar product of the two vectors $\sigma(\boldsymbol{x})$ and $\sigma(\boldsymbol{y})$ of $S^{1}$, the unit sphere in $\mathbb{R}^{2}$. The real function $J(|\boldsymbol{r}|)$ satisfies

$$
\begin{aligned}
& J(|\boldsymbol{r}|) \geqq 0 \quad \text { (ferromagnetism) } \\
& \sum_{\mathbf{0} \neq \boldsymbol{r} \in \mathbb{Z}^{2}} J(|\boldsymbol{r}|)<\infty
\end{aligned}
$$

and $h$ is a real constant. We define for the model I two different long-range order parameters:

$$
m_{0}=\lim _{h \rightarrow 0^{+}} \lim _{\Lambda \rightarrow \mathbb{Z}^{2}} 1 /|\Lambda|\left\langle\sum_{\boldsymbol{x} \in \Lambda} \sigma(\boldsymbol{x})\right\rangle_{h}
$$

where $\langle\cdot\rangle_{h}$ is the average taken with the hamiltonian (1), and

$$
m_{2}=\lim _{\Lambda \rightarrow \mathbb{Z}^{2}} 1 /|\Lambda|\left\langle\left|\sum_{\boldsymbol{x} \in \Lambda} \sigma(\boldsymbol{x})\right|^{2}\right\rangle^{-1 / 2} 0 .
$$

It is known that $m_{0} \geqq m_{2}[12]$. 
Our task is to show that there exists functions $J(r)$ and temperatures $T$ such that $m_{0}>0$. To solve this problem we proceed as in the paper of Dyson [9]: We construct a vectorial hierarchical model, called hereafter model II, for which we can prove

$$
0<m_{2}^{\mathrm{II}} \leqq m_{2}^{\mathrm{I}} \leqq m_{0}^{\mathrm{I}} .
$$

We describe this model [14] in the next section

\section{A Vectorial Hierarchical Model on $\mathbb{Z}^{2}$}

This model is also defined on $\mathbb{Z}^{2}$ and for his description we introduce the lexicographic order on $\mathbb{Z}^{2}$. Let us consider the square $\Lambda$ of $\mathbb{Z}^{2}$ whose smallest element is $(1,1)$ and which contains $4^{N}$ spins. We introduce all partitions of $\Lambda$, called hereafter levels, made out of $4^{N-p}$ square blocks of length $2^{p}$. These levels are labelled by the integers $p, 0 \leqq p \leqq N$, and we denote an element of the level $p$ by $\Lambda(p, \boldsymbol{x})$ :

$$
\Lambda(p, \boldsymbol{x})=\left\{\boldsymbol{y} \in \mathbb{Z}^{2} \mid\left(x_{1}-1\right) 2^{p}<y_{1} \leqq x_{1} 2^{p},\left(x_{2}-1\right) 2^{p}<y_{2} \leqq x_{2} 2^{p}\right\} .
$$

We remark that $2^{p} \boldsymbol{x}$ is the greatest point of $\Lambda(p, x)$ and that the natural length for the level $p$ is $2^{p}$. With each $\Lambda(p, x)$ we associate a block-spin $S_{\boldsymbol{x}}^{p}$,

$$
S_{\boldsymbol{x}}^{p}=\sum_{\boldsymbol{y} \in \Lambda(p, \boldsymbol{x})} \sigma(\boldsymbol{y}) .
$$

Each block of the level $p+1$ is built up by four adjacent blocks of the level $p$

$$
\Lambda(p+1, \boldsymbol{x})=\Lambda(p, \boldsymbol{x}) \cup \Lambda\left(p, \boldsymbol{x}+x_{1} \boldsymbol{e}_{1}\right) \cup \Lambda\left(p, \boldsymbol{x}+x_{2} \boldsymbol{e}_{2}\right) \cup \Lambda(p, 2 \boldsymbol{x}) .
$$

The hamiltonian for the level $p$ is

$$
H^{p}=-\left(1 / 4^{2 p+1}\right) b_{p+1} \sum_{r_{1}=1}^{2^{N-p-1}} \sum_{r_{2}=1}^{2^{N-p-1}} \sum_{\substack{i_{1}, j_{1}, i_{2}, j_{2}=0,1 \\\left(i_{1}, i_{2}\right)<\left(j_{1}, j_{2}\right)}} S_{2 r_{1-i_{1}, 2 r_{2}-i_{2}}}^{p} \cdot S_{2 r_{1}-j_{1}, 2 r_{2}-j_{2}}^{p}
$$

and the hamiltonian for the total system is

$$
H_{N}=\sum_{p=0}^{N-1} H^{p}
$$

In particular at the level $p$ two block-spins interact if and only if they are in the same block of the level $p+1$. Through the block-spins each spin interacts with other spins at each level, but two given spins interact only at a well-defined level. The $b_{p}$ are chosen positive.

\section{Existence of Long-Range Order for the Model II}

We give in this section a sufficient condition on the $b_{p}$ for the existence of longrange order, which is defined here by

with

$$
\left(m_{2}\right)^{2}=\lim _{\Lambda \rightarrow \infty} \sup \left(m_{2, N}\right)^{2}
$$

$$
\left(m_{2, N}\right)^{2}=1 / 4^{2 N}\left\langle\left(S_{(1,1)}^{N}\right)^{2}\right\rangle_{N} \equiv l_{N}
$$


where $\langle\cdot\rangle_{N}$ is the average taken in the model with $4^{N}$ spins. We establish first a relation between $l_{N}$ and $l_{N-1}$. Let $Y_{N}$ be the partition function and let us put $\boldsymbol{s}=S_{(1,2)}^{N-1}, \boldsymbol{t}=S_{(1,1)}^{N-1}, \boldsymbol{u}=S_{(2,1)}^{N-1}, \boldsymbol{v}=S_{(1,2)}^{N-1}$. We write $d \Omega=d \varphi$ for the measure on $S^{1}$ and form the expression

$$
Z_{N-1}(s)=\int \prod_{i} d \Omega_{i} e^{-\beta H_{N-1}} \delta\left(s-\sum_{\boldsymbol{x}} \sigma(\boldsymbol{x})\right), \quad \beta=1 / k T
$$

which is the partition function for fixed $s$ in the model II with $4^{N-1}$ spins. The invariance of rotation implies

$$
Z_{N-1}(s)=Z_{N-1}(s)
$$

where $s$ is the norm of $s$. We obtain easily the relation

$$
\begin{aligned}
l_{N} & =\left(1 / 4^{2 N}\right)\left\langle(\boldsymbol{s}+\boldsymbol{t}+\boldsymbol{u}+\boldsymbol{v})^{2}\right\rangle_{N} \\
& =\left(1 / 4^{2 N}\right)\left(4\left\langle\boldsymbol{s}^{2}\right\rangle_{N}+12\langle\boldsymbol{s} \cdot \boldsymbol{t}\rangle_{N}\right) \\
& \geqq(1 / 4) l_{N-1}+\left(3 / 4 \cdot 4^{2(N-1)}\right)\langle\boldsymbol{s} \cdot \boldsymbol{t}\rangle_{N}
\end{aligned}
$$

by applying the inequalities of Ginibre [11] which are analogous to those of Griffiths for the Ising model. If we apply once more these inequalities we find

$$
\langle\boldsymbol{s} \cdot \boldsymbol{t}\rangle_{N} \geqq\left\langle\int d \Omega e^{h s t \cos \varphi} s t \cos \varphi\right\rangle /\left\langle\int d \Omega e^{h s t \cos \varphi}\right\rangle
$$

with $h=\beta 4^{1-2 N} b_{N}$ and the average $\langle\cdot\rangle$ is taken with respect to the probability measure

$$
\left(Y_{N-1}\right)^{-2} Z_{N-1}(s) Z_{N-1}(t) s d s t d t .
$$

The reader may notice that this last estimate reduces the discussion of our model to the analogous one-dimensional hierarchical model. For this step it is essential to have inequalities like those of Ginibre.

The crucial step now is the proof of the following:

Lemma. If $\hat{x}=\left\langle(s t)^{2}\right\rangle^{1 / 2}$, then

$$
\left\langle\int d \Omega e^{h s t \cos \varphi} s t \cos \varphi\right\rangle /\left\langle\int d \Omega e^{h s t \cos \varphi}\right\rangle \geqq \hat{x}(1-1 / h \hat{x}) .
$$

Since the proof is a bit lengthy we postpone it to the end of the paper.

This result allows us to conclude if we remark that $\hat{x}=4^{2(N-1)} l_{N-1}$. Indeed we find

$$
\begin{aligned}
l_{N} & \geqq(1 / 4) l_{N-1}+(3 / 4) l_{N-1}\left(1-4 / l_{N-1} b_{N} \beta\right) \\
& =l_{N-1}-3 / b_{N} \beta,
\end{aligned}
$$

and by applying this recurrence relation $N$ times we prove the Theorem I.

Theorem I. The plane rotator hierarchical model with ferromagnetic interaction has a phase transition if

$$
B=\sum_{p \geqq 1} 1 / b_{p}<\infty .
$$

It has long-range order if $\beta>3 B$. 


\section{Existence of First Order Phase Transition for the Plane Rotator Model}

We compare here the interactions in the models I and II by means of Ginibre inequalities and we give a sufficient condition which ensures that $m_{2}^{\mathrm{II}} \leqq m_{2}^{\mathrm{I}}$. We restrict ourselves to the case where $J(\boldsymbol{r})=J(r)$ is a monotone decreasing positive function. We know that two spins in the model II interact only at a well-defined level. Furthermore if they interact at the level $p$ their relative distance $d$ satisfies

$$
1 \leqq d \leqq\left(2^{p+1}-1\right) \sqrt{2}<2^{p+1} \sqrt{2} .
$$

The desired condition is consequently

$$
J(r) \geqq \sup _{\substack{p \\ r<\sqrt{2} \cdot 2^{p+1}}} b_{p+1} / 4^{2 p+1}
$$

or

$$
J\left(\sqrt{2}\left(2^{p}-1\right)\right) \geqq \sup _{q \geqq p} b_{q} / 4^{2 q-1}
$$

because $J(r)$ is monotone decreasing.

Theorem II. The plane rotator model with ferromagnetic interaction and with $J(r)$ monotone decreasing undergoes a first order phase transition in two dimensions if

a) $\left(r^{5} J(r)\right)^{-1}$ is a monotone decreasing function and

b) $\sum_{h \in \mathbb{N}}\left(n^{5} J(n)\right)^{-1}<\infty$.

Corollary. If $J(r) \sim 1 / r^{\alpha}$, then the plane rotator model exhibits spontaneous magnetization at low temperature if $2<\alpha<4$.

Proof of the Theorem. We have spontaneous magnetization if

$$
\sum_{p \geqq 1} b_{p}^{-1}<\infty \text { with } \quad b_{p}=4^{2 p-1} J\left(\sqrt{2}\left(2^{p}-1\right)\right) \text {. }
$$

This condition is equivalent to

$$
\sum_{p \geqq 1} 2^{p}\left(2^{5 p} J\left(\sqrt{2}\left(2^{p}-1\right)\right)\right)^{-1}<\infty
$$

which is in turn equivalent to

$$
\sum_{n \in \mathbb{N}}\left(n^{5} J(n)\right)^{-1}<\infty
$$

if $n^{5} J(n)$ is a positive monotone decreasing function [13].

Proof of the Lemma. We prove here the fundamental lemma. This lemma was proved by Dyson when the $\sigma(x) \in S^{2}$ the unit sphere in $R^{3}$. We can prove the lemma when $\sigma(x) \in S^{n}$. Our main result does not extend however, because the inequalities of Ginibre are proven only for $n=1$. We divide the proof in four points.

A) We define first the function $f_{n}, n \geqq 2$

$$
f_{n}(x)=\left.\int_{-1}^{+1} d z\left(1-z^{2}\right)^{(n-3) / 2} z e^{x z}\right|_{-1} ^{+1} d z\left(1-z^{2}\right)^{(n-3) / 2} e^{x z}
$$

where $x \in \mathbb{R}^{+}$. 
This function has the properties

$$
f_{n}(0)=0, \quad f_{n}(x)=O(x) \text { and } f_{n}(x) \geqq 0 .
$$

It satisfies also the following differential equation

$$
(d / d x) f_{n}(x)=-f_{n}^{2}(x)+1-(n-1) x^{-1} f_{n}(x)
$$

which is easily derived if we note that

$$
\left(1-z^{2}\right)^{(n-3) / 2} z^{2} e^{x z}=\left(1-z^{2}\right)^{(n-3) / 2} e^{x z}-\left(1-z^{2}\right)^{(n-1) / 2} e^{x z}
$$

and

$$
\begin{aligned}
\int_{-1}^{+1}\left(1-z^{2}\right)^{(n-1) / 2} e^{x z} d z & =x^{-1} \int_{-1}^{+1}\left(1-z^{2}\right)^{(n-1) / 2}(d / d z) e^{x z} d z \\
& =(n-1) x^{-1} \int_{-1}^{+1}\left(1-z^{2}\right)^{(n-3) / 2} z e^{x z} d z
\end{aligned}
$$

B) The following inequality holds

$$
f_{n-1}(x) \geqq f_{n}(x), \quad n \geqq 2 .
$$

Proof. Let us consider the function $g_{n}=f_{n-1}-f_{n}$. We have $g(0)=0$ and

$$
(d / d x) g_{n}(x)=-\left(f_{n-1}(x)+f_{n}(x)\right) g_{n}(x)-(n-2) x^{-1} g_{n}(x)+x^{-1} f_{n}(x) .
$$

Let us suppose that there exists a positive $x_{1}$ such that $g_{n}\left(x_{1}\right) \leqq 0$. Then we must have $(d / d x) g_{n}\left(x_{1}\right)>0$. But this implies that $g_{n}(x)<g_{n}\left(x_{1}\right)$ for all $0<x<x_{1}$ and in particular $g_{n}(0) \leqq 0$ by continuity.

C) Let $v_{n}(x)$ be a probability measure and let us define for $\alpha \geqq 0$

and

$$
\varphi_{n}(\alpha)=\frac{\int_{0}^{\infty} d x v_{n}(x) x \int_{-1}^{+1} d z\left(1-z^{2}\right)^{(n-3) / 2} z e^{\alpha x z}}{\int_{0}^{\infty} d x v_{n}(x) \int_{-1}^{+1} d z\left(1-z^{2}\right)^{(n-3) / 2} e^{\alpha x z}}
$$

$$
\hat{x}_{n}^{2}=\int_{0}^{\infty} d x v_{n}(x) x^{2} / \int_{0}^{\infty} d x v_{n}(x) .
$$

Lemma. $\varphi_{n}(\alpha) \geqq \hat{x}_{n} f_{n}\left(\alpha \hat{x}_{n}\right)$.

Proof. As before we derive the following differential equation for $\varphi_{n}(\alpha)$ :

$$
(d / d \alpha) \varphi_{n}(\alpha)=-\varphi_{n}^{2}(\alpha)-(n-1) \alpha^{-1} \varphi_{n}(\alpha)+\left\langle x^{2}\right\rangle_{n}(\alpha)
$$
with

$$
\left\langle x^{2}\right\rangle_{n}(\alpha)=\frac{\int_{0}^{\infty} d x v_{n}(x) x^{2} I_{n}(\alpha x)}{\int_{0}^{\infty} d x v_{n}(x) I_{n}(\alpha x)}=\frac{\left\langle x^{2} I_{n}(\alpha x)\right\rangle_{v_{n}}}{\left\langle I_{n}(\alpha x)\right\rangle_{v_{n}}}
$$

and

$$
I_{n}(\alpha x)=\int_{-1}^{+1} d z\left(1-z^{2}\right)^{(n-3) / 2} e^{\alpha x z}
$$


We see easily that $I_{n}(\alpha x)$ is increasing with $x$ and thus we obtain by Tchebychef's inequality

$$
\left\langle x^{2}\right\rangle_{n}(\alpha) \geqq\left\langle x^{2}\right\rangle_{v_{n}}=\hat{x}_{n}^{2} .
$$

Consequently we have for the derivative of $\varphi_{n}(\alpha)$ with respect to $\alpha$

$$
(d / d \alpha) \varphi_{n}(\alpha) \geqq-\varphi_{n}^{2}(\alpha)-(n-1) \alpha^{-1} \varphi_{n}(\alpha)+\hat{x}_{n}^{2} .
$$

Furthermore $\varphi_{n}(0)=0$ and $\varphi_{n}(\alpha) \geqq 0$. Let $\psi_{n}(\alpha)$ be the solution of

$$
d \psi_{n} / d \alpha=-\psi_{n}^{2}-(n-1) \alpha^{-1} \psi_{n}+\hat{x}_{n}^{2} .
$$

Then we find immediately that

$$
\psi_{n}(\alpha)=\hat{x}_{n} f_{n}\left(\alpha \hat{x}_{n}\right)
$$

and by the same argument as in B)

$\varphi_{n} \geqq \psi_{n}$.

D) It is easy to calculate $f_{3}(x)$,

$$
f_{3}(x)=\operatorname{cth}(x)-1 / x \geqq 1-1 / x .
$$

This implies in particular that

$\varphi_{2}(\alpha) \geqq \hat{x}_{2}\left(1-1 / \alpha \hat{x}_{2}\right)$.

\section{References}

1. Mermin, N. D., Wagner, H.: Phys. Rev. Letter 17, 1133 (1966)

2. Ruelle, D.: Statistical Mechanics, p. 131. New York: Benjamin 1969

3. Mermin, N.D.: J. Math. Phys. 8, 1061 (1967)

4. Vuillermot, P.-A., Romério, M. V.: Commun. math. Phys. 41, 281 (1975)

5. Dobrushin, R.L., Shlosman, S. B.: Commun. math. Phys. 42, 31--40 (1975)

6. Fisher, M.E.: J. Appl. Phys. 38, 981 (1966)

7. Mermin, N. D.: J. Phys. Soc. Japan 26 (Suppl.), 203 (1969)

8. Thouless, D. J.: Phys. Rev. 187, 732 (1969)

9. Dyson, F. J.: Commun. math. Phys. 12, 91 (1969)

10. Dyson, F. J.: Commun. math. Phys. 21, 269 (1971)

11. Ginibre, J.: Commun. math. Phys. 16, 310 (1970)

12. Griffiths, R. B.: Phys. Rev. 152, 240 (1966)

13. Knopp, K.: Theorie und Anwendung der unendlichen Reihen, chap. 3. Berlin-HeidelbergNew York: Springer 1964

14. Baker, A.: Phys. Rev. 135, 2622 (1972)

Communicated by G. Gallavotti

Received July 10, 1975

Note Added in Proof. It is possible to show, by means of Bogoliubov inequality, that there is no spontaneous magnetization when $\alpha=4$. 
